\title{
versants
}

REVUE SUISSE DES LITTÉRATURES ROMANES

Revue publiée sous les auspices du Collegium Romanicum

(Association des romanistes suisses) avec le concours de l'Académie suisse

des sciences humaines et sociales

NUMÉRO 63:1 (FASCICULE FRANÇAIS)

2016

\section{À QUOI BON L'ENSEIGNEMENT DE LA LITTÉRATURE?}

Sous la direction d'Ursula Bähler et de Thomas Klinkert

\section{SLATKINE}

GENÈVE

Diffusion en France:

HONORÉ CHAMPION ÉDITEUR,

Paris 

des sciences humaines et sociales www.assh.ch

(C) 2016. Éditions Slatkine, Genève.

www.slatkine.com

Reproduction et traduction, même partielles, interdites.

Tous droits réservés pour tous les pays.

ISBN 978-2-05-102789-2 ISBN 978-2-05-102792-2

ISSN 0256-9645 


\section{Lire une partition}

\section{Mon rêve}

Je rêve d'un pays où des centaines et des milliers de jeunes et de moins jeunes suivraient des cours de lecture. Dans leur temps libre. Sans contrainte. Ils paieraient les droits d'inscription, sans se poser la question de l'utilité de leur dépense. Leur apprentissage ne leur apporterait aucun avantage matériel, aucun avancement professionnel. Leur but ne serait pas de devenir des écrivains. Enthousiastes et têtus, ils s'appliqueraient néanmoins à lire des textes littéraires de plus en plus difficiles. Ils liraient et reliraient des pages et des pages, en silence ou à haute voix, écouteraient des explications, méditeraient les passages obscurs, discuteraient en groupe, pour vivre, enfin, le moment magique où, dans l'acte de lecture, son et rythme, parole et signification, sous-entendus et renvois forment un tout qui n'est plus lettre morte mais expérience vécue. Certes, il arrive qu'une lecture touche au but du premier coup, comme un air qu'on exécute au piano, sans réfléchir, sans trébucher, sans dissonance. Le plus souvent, cependant, les apprentis lecteurs ne connaissent la lumière, l'harmonie et la jouissance du texte qu'au bout d'un exercice assidu.

Les habitants du pays dont je rêve ont l'air heureux. Chacun fait «ce que voudra ". Et chacune. Personne n'est contraint de suivre des cours de lecture. Ils et elles sont libres de perfectionner d'autres talents: le chant, le dessin, la danse, le sport... Rares sont ceux ou celles qui ne font rien du tout: le plaisir de maitriser un art vaut bien l'effort de s'y appliquer.

\section{La réalité}

Je me réveille dans un pays qui enseigne la lecture à la maternelle, à l'école primaire, au lycée et à l'université comme une compétence élémentaire, nécessaire, voire indispensable pour la vie sociale. Il faut bien que les citoyens soient suffisamment alphabétisés pour s'informer dans un journal (à défaut d'écouter la radio ou de s'enquérir des actualités à la télévision et sur Internet), pour déchiffrer correctement le mode 
d'emploi d'un appareil, la notice d'emballage d'un médicament, une recette de cuisine ou pour comprendre, à un niveau supérieur, des textes administratifs, juridiques, politiques, voire scientifiques. Que cette compétence, à l'occasion, puisse servir à lire un roman ou un poème, à condition que ces textes littéraires ne découragent pas, par un excès d'artifice et de difficulté, est envisagé comme un bénéfice secondaire. Dans le même temps, ce pays - le pays de Markus Hirscher et de Wolfgang Amadeus Mozart - trouve absolument normal que ses fils... et ses filles (pour citer correctement l'hymne national) s'entraînent, par milliers, dans des clubs de foot ou des écoles de ski et qu'ils et elles ne se contentent pas du b.a.ba de l'enseignement musical, dispensé à l'école, mais apprennent, eux et elles aussi, parce que ce ne sont pas toujours les mêmes, à jouer des instruments de musique pour exécuter tant bien que mal la Marche turque ou intégrer un orchestre folklorique.

Comment se fait-il que nos sociétés occidentales reconnaissent au chant, au dessin, à la danse, etc., mais aussi à certaines habiletés sportives le prestige de l'art, alors que la lecture n'a rang que de simple compétence? Comment se fait-il que tout le monde accepte que les premières activités demandent un perfectionnement de longue haleine, alors que la dernière est censée s'apprendre, sans effort supplémentaire, durant les heures de classe?

\section{Un autre parallèle des arts}

On pourrait m'objecter que la lecture relève d'une capacité essentiellement réceptive, n'ayant rien à voir avec l'acte créateur de l'artiste qui peint un portrait ou un paysage, de la soprano qui chante un solo d'opéra, du pianiste qui joue la Sonate au clair de lune ou - pourquoi pas? - du skieur de descente qui trouve miraculeusement la ligne idéale. Mais tous ces créateurs n'ont-ils pas, eux aussi, un canevas dans la tête ou devant les yeux qu'ils suivent librement, en cherchant à approcher la ligne idéale de l'interprétation individuelle qui définira leur personnalité d'artiste? Estce l'utopie d'atteindre - une fois, de temps en temps ou de plus en plus souvent - la réalisation parfaite, à la fois correcte et personnelle, d'un programme artistique, qui motive des milliers de jeunes et de moins jeunes à s'exercer, à répéter des mouvements maladroits jusqu'à la nausée, 
à accepter les corrections, à vaincre les défaites, à persévérer avec acharnement? Tous ces efforts pour vivre un moment de grâce?

Le texte littéraire n'est-il pas, lui aussi, une partition qui ne prend corps que par l'acte de lecture? La lecture est un art au même rang que les autres. L'art de transformer les lettres mortes - noires - qui dansent devant nos yeux en images intérieures. L'art d'accéder au monde évoqué par les paroles d'un auteur, de l'habiter pendant le temps de la lecture, en prenant nos aises avec ce qui parait curieux, étrange, blâmable, impossible ou simplement difficile, en acceptant, en respectant l'altérité de ce monde sans le quitter par la porte de l'improvisation libre. Le Combray qu'ima-

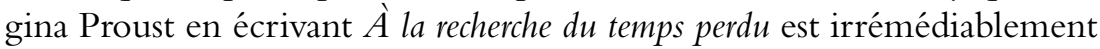
perdu, mais chaque lecteur, chaque lectrice de Proust est appelé à créer une image singulière de ce lieu mythique dans sa tête, quoique tous et toutes s'appuient sur la même partition.

Pratiquer l'art de la lecture, à l'université ou ailleurs, ce serait chercher et trouver ensemble, enseignants et apprentis lecteurs confondus, les règles (linguistiques, rhétoriques, historiques, psychologiques et autres) qui permettent de jouer le jeu de l'auteur et d'avancer dans l'exploration de tel monde spécifique, symbolisé par tel assemblage de lettres, mais en le recréant, à chaque fois, en son for intérieur, à sa façon, selon sa sensibilité, son savoir et ses valeurs. N'importe quel texte littéraire demande, telle une partition musicale, le respect des contraintes qui limitent les marges de manœuvre de l'exécutant. Enseigner l'art de la lecture, c'est donc apprendre à découvrir dans la forme-sens qu'est tout texte littéraire les potentialités d'une lecture active (recréative et récréative), toujours individuelle.

Mais la lecture, dans le pays que j'habite, n'est qu'une compétence... et mon rêve persiste.

Peter KUON

Universität Salzburg 